The Annals of Probability

1989, Vol. 17, No. 4, 1416-1431

\title{
UNIQUENESS OF GIBBS MEASURES AND ABSORPTION PROBABILITIES
}

\author{
By HENRY BERBEe \\ Centre for Mathematics and Computer Science, Amsterdam
}

Gibbs measures are studied using a Markov chain on the nonnegative integers. Uniqueness of Gibbs measures follows from absorption of the chain at $\{0\}$. To this end, we derive a certain inequality. For one-dimensional systems this extends a well-known uniqueness result of Ruelle and for models near the $1 / r^{2}$-interaction Ising model it is a natural improvement of some other results.

1. Introduction. In studying uniqueness of Gibbs measures it may be useful to make a comparison with a stochastic process. We present a method connecting absorption probabilities of a simple Markov chain at $\{0\}$ with uniqueness. Our specific result, Theorem 1.1, is used for one-dimensional systems. It includes a well-known result on uniqueness in Ruelle (1968) and succeeds in Section 6 to find a natural improvement of other results that needed refined estimates. In our method the key role is played by a certain inequality. Comparison of processes is quite well known already in the literature: Duality [see Liggett (1985)] is a nice technique based on an equality. We hope that our comparison method using absorption probabilities is useful in more problems.

Let us now describe our problem setting. Let $S$ be a countable set and $X$ a finite or countable set. For $\Lambda \subset S$ and $\sigma \in X^{S}$ write $\sigma_{\Lambda}=\left(\sigma_{j}\right)_{j \in \Lambda}$. We define Gibbs measures on $X^{S}$ in terms of a given energy difference function $\Delta H\left(\sigma, \sigma^{\prime}\right)$ for the energy difference between $\sigma$ and $\sigma^{\prime}$ in $X^{S}$. This function has to be properly defined only in case $\sigma_{\bar{\Lambda}}=\sigma^{\prime}{ }_{\bar{\Lambda}}$ for the complement $\bar{\Lambda}$ of any finite set $\Lambda$. Assume that for any finite $\Lambda \subset S$ this energy difference has the form

$$
\Delta H\left(\sigma, \sigma^{\prime}\right)=\phi_{\Lambda}(\sigma)-\phi_{\Lambda}\left(\sigma^{\prime}\right) \text { if } \sigma_{\bar{\Lambda}}=\sigma^{\prime}{ }_{\Lambda},
$$

where $\phi_{\Lambda}$ is a suitable real function on $X^{S}$. Let us define a probability measure $\rho_{\Lambda, \sigma}$ on $X^{\Lambda}$ by

$$
\rho_{\Lambda, \sigma}\left(\left\{\sigma^{\prime}{ }_{\Lambda}\right\}\right):=\frac{1}{Z_{\Lambda, \sigma}} \exp \left(-\phi_{\Lambda}\left(\sigma^{\prime}\right)\right)
$$

where

$$
Z_{\Lambda, \sigma}:=\sum_{\sigma_{\Lambda}^{\prime} \in X^{\Lambda}} \exp \left(-\phi_{\Lambda}\left(\sigma^{\prime}\right)\right) \text { with } \sigma^{\prime}{ }_{\bar{\Lambda}}=\sigma_{\bar{\Lambda}} .
$$

Clearly $\rho_{\Lambda, \sigma}$ as a function of $\sigma$ does not depend on $\sigma_{\Lambda}$ and moreover it is the

Received February 1987; revised November 1988.

AMS 1980 subject classifications. Primary 82A25; secondary 47D45, 60K35.

Key words and phrases. Uniqueness of Gibbs measures, positive operator, Markov operator, duality, absorbing state, inequality, Perron-Frobenius theorem. 
probability measure such that

$$
\frac{\rho_{\Lambda, \sigma}\left(\left\{\sigma_{\Lambda}\right\}\right)}{\rho_{\Lambda, \sigma^{\prime}}\left(\left\{\sigma_{\Lambda}^{\prime}\right\}\right)}=\exp \left(-\Delta H\left(\sigma, \sigma^{\prime}\right)\right) \text { if } \sigma_{\bar{\Lambda}}=\sigma^{\prime}{ }_{\bar{\Lambda}} .
$$

We call a probability measure $\mu$ on $X^{S}$ a Gibbs measure for $\Delta H$ if for any finite $\Lambda \subset S$,

$$
\mu\left(d \sigma_{\Lambda}, d \sigma_{\bar{\Lambda}}\right)=\rho_{\Lambda, \sigma}\left(d \sigma_{\Lambda}\right) \mu_{\bar{\Lambda}}\left(d \sigma_{\bar{\Lambda}}\right),
$$

where $\mu_{\bar{\Lambda}}$ is the restriction of $\mu$ to $X^{\bar{\Lambda}}$. Note that if (1.1) holds for $\Lambda$, then it holds also for $\tilde{\Lambda} \subset \Lambda$.

Section 6 contains an illustration of the notation and definitions above for a one-dimensional long range interaction Ising model.

We will describe now the context of the main theorem. Suppose there are given finite sets $\varnothing=\Lambda_{0} \subset \Lambda_{1} \subset \cdots \uparrow S$. Write

$$
\sigma_{n}:=\sigma_{\Lambda_{n} \backslash \Lambda_{n-1}}, \quad n \geq 1,
$$

and define

$$
\phi_{n}(\sigma)=\phi_{\Lambda_{n}}(\sigma)-\phi_{\Lambda_{n-1}}(\sigma), \quad n \geq 1 .
$$

It is easily checked that the function $\phi_{n}$ does not depend on $\sigma_{1}, \ldots, \sigma_{n-1}$ and so we may write

$$
\phi_{n}(\sigma)=\phi_{n}\left(\sigma_{n}, \sigma_{n+1}, \ldots\right) \text {. }
$$

Define for $k \geq 0$,

$$
\begin{aligned}
\operatorname{var}_{k}\left(\phi_{n}\right) & :=\max _{\sigma_{n}, \ldots, \sigma_{n+k}}\left[\bar{\phi}_{n}\left(\sigma_{n}, \ldots, \sigma_{n+k}\right)-\underline{\phi}_{n}\left(\sigma_{n}, \ldots, \sigma_{n+k}\right)\right], \\
r_{k} & :=\operatorname{supvar}_{n \geq 1}\left(\phi_{n}\right),
\end{aligned}
$$

where

$$
\begin{aligned}
& \bar{\phi}_{n}\left(\sigma_{n}, \ldots, \sigma_{n+k}\right):=\sup _{\left(\sigma_{j}\right)_{j>n+k}} \phi_{n}\left(\sigma_{n}, \sigma_{n+1}, \ldots\right), \\
& \phi_{n}\left(\sigma_{n}, \ldots, \sigma_{n+k}\right):=\inf _{\left(\sigma_{j}\right)_{j>n+k}} \phi_{n}\left(\sigma_{n}, \sigma_{n+1}, \ldots\right) .
\end{aligned}
$$

We assume that $\operatorname{var}_{k}\left(\phi_{n}\right) \downarrow 0$ as $k \rightarrow \infty$ for any $n \geq 1$.

Related to the result of Ruelle (1968) for uniqueness of Gibbs states is the condition

$$
\sum_{k \geq 0} r_{k}<\infty
$$

Our theorem below uses the weaker condition (1.3).

THEOREM 1.1. There exists at most one Gibbs measure for $\Delta H$ if

$$
\sum_{k \geq 0} \exp \left(-r_{0}-\cdots-r_{k}\right)=\infty
$$


It may happen that $r_{0}=\infty$ if, e.g., $S=\mathbb{Z}^{d}$ is higher dimensional. Note also that the result is temperature sensitive. Suppose, e.g., that $\Delta H_{\beta}\left(\sigma, \sigma^{\prime}\right)=$ $\beta \Delta H\left(\sigma, \sigma^{\prime}\right)$. One obtains a bound on the critical inverse temperature from the theorem by using the following lemma.

LEMMA 1.2.

$$
\sum_{k \geq 0} \exp \left(-\beta\left(r_{0}+\cdots+r_{k}\right)\right)=\infty \text { for } \beta<\beta_{*}:=\liminf \frac{\log k}{r_{0}+\cdots+r_{k}} .
$$

We leave the proof to the reader (see also Section 6). The example in Hofbauer (1977) is related to the form of our result. For chains with infinite connections Berbee (1987) gives a uniqueness result under similar conditions but with a quite different proof. In Remark 4.1 we indicate a relation using duality.

REMARK 1.3. In Section 3 it will follow that Theorem 1.1 remains valid if we let $N$ be any positive integer and replace $r_{k}$ in (1.3) by

$$
r_{k}^{\prime}:=\sup _{n \geq N} \operatorname{var}_{k}\left(\phi_{n}\right)
$$

This is important to understand the performance of our result: It follows that individual $\phi_{n}$ essentially play no role in this way in our uniqueness criterion.

Our approach consists of an analysis of (1.1) using positive operators. This can be explained as follows. Note that $Z_{\Lambda, \sigma}$ above depends only on $\sigma_{\bar{\Lambda}}$. Thus the measure

$$
\mu_{n}:=\frac{1}{Z_{\Lambda_{n}, .}} \mu_{\bar{\Lambda}_{n}}
$$

on $X^{\bar{\Lambda}_{n}}$ is properly defined. We investigate the operators $L_{n}$ for which

$$
L_{n} \mu_{n}=\mu_{n-1}, \quad n \geq 1, \mu_{0} \equiv \mu .
$$

In Section 2 we construct a Markov matrix related to the operator $L_{n}$. Based on our approach in Section 2 we obtain in Section 3 a general inequality that is our key result and is formulated using absorption probabilities for a Markov chain. As a corollary we get a certain uniqueness result for Gibbs measures and an inequality for correlations. In Section 4 we use this to get Theorem 1.1. In Section 5 we prove a result in Perron-Frobenius theory and indicate how our result differs from Ruelle (1968). Section 6 discusses our Ising model application.

2. Ratio bounds. We define ratio bounds of certain measures and investigate their behavior under the application of positive operators $L$ on these measures. This leads us at the end of the section to associate a Markov operator with $L$.

Let $Y:=\Pi_{k \geq 1} Y_{k}$ where $Y_{1}, Y_{2}, \ldots$ are finite sets. Let $\mu, \nu \in \mathscr{M}(Y)$ with $\mathscr{M}(Y)$ the space of bounded measures on the space $Y$. We compare $\mu$ with $\nu$ using "ratio bounds" that are defined as follows, using the product structure of Y. Let 
$\mathscr{C}_{k}$ consist of the $k$-cylinder sets $C \subset Y$ having the form

$$
C=A \times Y_{k+1} \times Y_{k+2} \times \cdots \quad \text { where } A \subset Y_{1} \times \cdots \times Y_{k} .
$$

The ratio bounds for the measure $\mu$ with respect to $\nu$ on $Y$ are the coefficients

$$
\begin{aligned}
\rho_{k} & :=\sup _{C \in \mathscr{C}_{k}} \frac{\mu(C)}{\nu(C)}, \\
\rho_{-k} & :=\inf _{C \in \mathscr{C}_{k}} \frac{\mu(C)}{\nu(C)}, \quad k \geq 0 .
\end{aligned}
$$

Note that $\rho_{k}=\rho_{k}(\mu, \nu)$ satisfies

$$
\rho_{k}(\mu, \nu)=\rho_{-k}(\nu, \mu)^{-1} .
$$

We also have $\rho_{0}(\mu, \nu)=\|\mu\| /\|\nu\|$.

Let us now consider a sequence $X_{1}, X_{2}, \ldots$ of finite or countable sets and define $X_{(n)}:=\prod_{k \geq 1} X_{n+k}, n \geq 0$. Fixing $n \geq 1$ we define an operator $L \equiv \exp (\phi)$ such that $L: \mathscr{M}\left(X_{(n)}\right) \rightarrow \mathscr{M}\left(X_{(n-1)}\right)$ as follows. Let $\phi \equiv \phi_{n}$ be a real function on $X_{(n-1)}$ and define $L \equiv L_{n}$ by

$$
L \mu(B):=\sum_{\sigma_{n} \in X_{n}} \int_{X_{(n)}} \exp \left(\phi\left(\sigma_{n}, x\right)\right) 1_{B}\left(\sigma_{n}, x\right) \mu(d x)
$$

for $\mu \in \mathscr{M}\left(X_{(n)}\right)$. For $\mu, \nu \in \mathscr{M}\left(X_{(n)}\right)$ we write

$$
\tilde{\rho}_{k}(\mu, \nu):=\rho_{k}(L \mu, L \nu) .
$$

Writing $\phi$ as $\phi_{n}\left(\sigma_{n}, \sigma_{n+1}, \ldots\right)$ with $\sigma_{j} \in X_{j}$ we have, using the definition of $\operatorname{var}_{k} \phi$ in the preceding section:

Proposition 2.1. For $1 \leq k \leq N$ with any $N$

$$
\begin{gathered}
\tilde{\rho}_{-k} \geq \rho_{-(k-1)} \exp \left(-\operatorname{var}_{k-1} \phi\right)+\rho_{-k}\left(\exp \left(-\operatorname{var}_{k} \phi\right)-\exp \left(-\operatorname{var}_{k-1} \phi\right)\right) \\
+\cdots+\rho_{-N}\left(\exp \left(-\operatorname{var}_{N} \phi\right)-\exp \left(-\operatorname{var}_{N-1} \phi\right)\right) .
\end{gathered}
$$

Proof. Write $\left[\sigma_{n}, \ldots, \sigma_{n+k-1}\right]_{n}$ for the cylinder $C \subset X_{(n-1)}$ of the form

$$
C=\left\{\sigma_{n}\right\} \times\left\{\sigma_{n+1}\right\} \times \cdots \times\left\{\sigma_{n+k-1}\right\} \times \prod_{j \geq n+k} X_{j} .
$$

We want to bound $L \mu(C) / L \nu(C)$ and decompose $L \mu(C)$ as

$$
\begin{aligned}
& \int \exp \left(\underline{\phi}\left(\sigma_{n}, \ldots, \sigma_{n+k-1}\right)\right) 1_{C}\left(\sigma_{n}, x\right) \mu(d x) \\
& \quad+\sum_{k \leq m \leq N} \int\left(\exp \left(\underline{\phi}\left(\sigma_{n}, \ldots, \sigma_{n+m}\right)\right)-\exp \left(\underline{\phi}\left(\sigma_{n}, \ldots, \sigma_{n+m-1}\right)\right)\right) 1_{C}\left(\sigma_{n}, x\right) \mu(d x) \\
& \quad+\int\left(\exp \left(\phi\left(\sigma_{n}, x\right)\right)-\exp \left(\underline{\phi}\left(\sigma_{n}, \ldots, \sigma_{n+N}\right)\right)\right) 1_{C}\left(\sigma_{n}, x\right) \mu(d x) .
\end{aligned}
$$


Here the integration is over $X_{(n)}$. We use, after decomposing $C$ in smaller cylinders,

$$
\mu\left(\left[\sigma_{n+1}, \ldots, \sigma_{n+m}\right]_{n+1}\right) \geq \rho_{-m} p\left(\left[\sigma_{n+1}, \ldots, \sigma_{n+m}\right]_{n+1}\right) .
$$

This gives

$$
\begin{aligned}
L \mu(C) \geq \int\left\{\rho_{-(k-1)} \exp \left(\underline{\phi}\left(\sigma_{n}, \ldots, \sigma_{n+k-1}\right)\right)\right. \\
+\sum_{k \leq m \leq N} \rho_{-m}\left(\exp \left(\underline{\phi}\left(\sigma_{n}, \ldots, \sigma_{n+m}\right)\right)\right. \\
\left.\left.\quad-\exp \left(\underline{\phi}\left(\sigma_{n}, \ldots, \sigma_{n+m-1}\right)\right)\right)+0\right\} 1_{C}\left(\sigma_{n}, x\right) \nu(d x) .
\end{aligned}
$$

We rearrange the term between $\{\cdot\}$ as

$$
\begin{aligned}
& \left(\rho_{-(k-1)}-\rho_{-k}\right) \exp \left(\underline{\phi}\left(\sigma_{n}, \ldots, \sigma_{n+k-1}\right)\right) \\
& \quad+\cdots+\left(\left(\rho_{-(N-1)}\right)-\rho_{-N}\right) \exp \left(\underline{\phi}\left(\sigma_{n}, \ldots, \sigma_{n+N-1}\right)\right) \\
& \quad+\rho_{-N} \exp \left(\underline{\phi}\left(\sigma_{n}, \ldots, \sigma_{n+N}\right)\right) .
\end{aligned}
$$

Because $\rho_{-j}$ is nonincreasing in $j$ the terms $(\cdot)$ are nonnegative. It is easily seen that

$$
\exp \left(\underline{\phi}\left(\sigma_{n}, \ldots, \sigma_{n+m}\right)\right) \geq \exp \left(-\operatorname{var}_{m}(\phi)\right) \exp \left(\phi\left(\sigma_{n}, x\right)\right),
$$

where $x=\left(\sigma_{n+1}, \sigma_{n+2}, \ldots\right)$. We apply this to (2.3) and rearrange again. Finally we get $L \mu(C) \geq \psi L \nu(C)$ where $\psi$ is the right-hand side in (2.2). Because $C$ is any $k$-cylinder set this implies the assertion on $\tilde{\rho}_{-k}$.

Proposition 2.1 is formulated for finite $N$. We may let $N \rightarrow \infty$. The result thus obtained will be summarized in (2.4) using a Markov transition matrix $P$.

We think of $P$ as being associated with the operator $L$, defined as follows. Let $\{0\}$ be an absorbing state for $P$ by writing $P_{00}=1$. Define a probability measure $F$ on $\{0,1,2, \ldots\}$ by letting

$$
F(k, \infty)=1-\exp \left(-\operatorname{var}_{k}(\phi)\right), \quad k \geq 0 .
$$

Because we assume $\operatorname{var}_{k}(\phi) \downarrow 0$ as $k \rightarrow \infty$, the measure $F$ is a probability measure on $\{0,1,2, \ldots\}$ and we let this be the state space associated with the Markov matrix. Now define for $1 \leq k<\infty$,

$$
\begin{aligned}
P_{k j} & :=F[0, k-1], & & \text { if } j=k-1 \geq 0, \\
& :=F\{j\}, & & \text { if } j \geq k .
\end{aligned}
$$

Thus the Markov matrix $P$, having row sums equal to 1 , is finally determined. From Proposition 2.1 we have the lower bound

$$
\tilde{\rho}_{-k} \geq \sum_{0 \leq j<\infty} P_{k j} \rho_{-j}, \quad k \geq 1 .
$$


This formula, giving a lower bound for $\tilde{\rho}$ in terms of the Markov matrix $P$ working on $\rho$, will be iterated in Section 3 to give our Lemma 3.1. Note that $k=0$ is not mentioned in (2.4).

3. An inequality and a Markov chain absorbed at $\{0\}$. We take again the point of view of Section 1 and connect it with Section 2 in the proof of key Lemma 3.1. There are given finite sets $\varnothing=\Lambda_{0} \subset \Lambda_{1} \subset \cdots \uparrow S$. Write $X_{(n)}:=$ $X^{\bar{\Lambda}_{n}}$. Note that $\phi_{n}=\phi_{n}\left(\sigma_{n}, \sigma_{n+1}, \ldots\right)$ defined by (1.2) can be identified with a function on $X_{(n-1)}$. Let $Z_{n}, n \geq 0$, be a Markov chain with the nonhomogeneous transition probabilities $P^{(1)}, P^{(2)}, \ldots$ where $P^{(n)}$ is the Markov transition matrix associated at the end of Section 2 with the operator $L_{n}:=\exp \left(\phi_{n}\right)$.

Let $N \geq 1$ and suppose $\mu$ is a probability measure on $X^{S}$ that is "right" on $\Lambda_{N}$ for $\Delta H$ in the sense that (1.1) holds for $\Lambda=\Lambda_{N}$. Assume the probability measure $\nu$ is also "right" in this sense. Clearly this is valid if $\mu$ and $\nu$ are Gibbs measures for $\Delta H$. Define for the Markov chain $Z_{n}, n \geq 0$, the absorption time at $\{0\}$ as

$$
\tau:=\inf \left\{n \geq 0: Z_{n}=0\right\}
$$

and let $P_{k}(\cdot):=P\left(\cdot \mid Z_{0}=k\right)$ be the conditional probability given that the chain is started at $\{k\}$. Nice monotonicity properties of this chain are discussed in the proof of Proposition 3.3. First we state our key inequality.

LEMMA 3.1. If $\mu$ and $\nu$ are probability measures that are "right" on $\Lambda_{N}$ for $\Delta H$ in the sense above, then for any $k \geq 1$,

$$
\inf _{C \in \mathscr{D}_{\Lambda_{k}}} \frac{\mu(C)}{\nu(C)} \geq P_{k}(\tau \leq N)^{2}
$$

Here $\mathscr{B}_{\Lambda}$ are the sets in $X^{S}$ generated by the projection on $X^{\Lambda}$.

Corollary 3.2. Suppose for all $k \geq 1$,

$$
P_{k}\left(Z_{n} \text { is absorbed at }\{0\}\right)=1 \text {. }
$$

Then there exists at most one Gibbs measure for $\Delta H$.

Let us mention that (3.2) can be weakened. Individual transition probabilities do not play an important role in its validity because it is sufficient that (3.2) holds for one of the chains $Z_{n}^{\prime}=Z_{n+N}, n \geq 1$, where $N$ is any positive integer. Moreover (3.2) has to be checked generally only for $k=1$ as can be seen in Proposition 3.3.

Proof of Corollary 3.2. If $\mu$ and $\nu$ are Gibbs measures, then we can apply the lemma and the right-hand side of (3.1) is asymptotically 1 for any $k$. Hence $\mu(C) \geq \nu(C)$ for any such $\mu$ and $\nu$ and any cylinder set $C$. This implies uniqueness of $\mu$. 
Proof of Lemma 3.1. Note that if $\mu_{n}$ is defined for $0 \leq n \leq N$ by (1.4), then defining $L_{n}:=\exp \left(\phi_{n}\right)$ as in Section 2 we have

$$
\mu \equiv \mu_{0}=L_{1} \cdots L_{n} \mu_{n}, \quad 1 \leq n \leq N .
$$

We prove by induction that for $n=N, N-1, \ldots, 1,0$,

$$
R_{k}(n):=\left(\frac{\rho_{-k}\left(\mu_{n}, \nu_{n}\right)}{\rho_{k}\left(\mu_{n}, \nu_{n}\right)}\right)^{1 / 2} \geq P\left(Z_{N}=0 \mid Z_{n}=k\right), \quad k \geq 0 .
$$

For $k=0$ and also $n=N$ this is trivial. For $k \geq 1$ and any $N \geq n>0$ we have (2.4) and similarly after using (2.1),

$$
\frac{1}{\rho_{k}\left(\mu_{n-1}, \nu_{n-1}\right)} \geq \sum_{0 \leq j<\infty} P_{k j}^{(n)} \frac{1}{\rho_{j}\left(\mu_{n}, \nu_{n}\right)}
$$

Using this in

$$
\left(\sum_{j} P_{k j} x_{j}\right)^{1 / 2}\left(\sum_{j} P_{k j} \frac{1}{y_{j}}\right)^{1 / 2} \geq \sum_{j} P_{k j}\left(\frac{x_{j}}{y_{j}}\right)^{1 / 2}
$$

which is a consequence of Cauchy's inequality, we find

$$
R_{k}(n-1) \geq \sum_{j} P_{k, j}^{(n)} R_{j}(n) \geq \sum_{j} P_{k, j}^{(n)} P\left(Z_{N}=0 \mid Z_{n}=j\right)=P\left(Z_{N}=0 \mid Z_{n-1}=k\right)
$$

using induction. This proves the induction step. Now observe

$$
\left(\frac{\rho_{-k}(\mu, \nu)}{\rho_{k}(\mu, \nu)}\right)^{1 / 2}=R_{k}(0) \geq P\left(Z_{N}=0 \mid Z_{0}=k\right)=P_{k}(\tau \leq N) .
$$

Because

$$
\rho_{k}(\mu, \nu) \geq \rho_{0}(\mu, \nu)=\frac{\|\mu\|}{\|\nu\|}=1,
$$

this implies the assertion.

Quite generally the condition of the corollary can be relaxed by the following proposition.

Proposition 3.3. Suppose $P_{1}\left(Z_{k} \neq 0\right)>0$ for all $k \geq 1$. Then (3.2) holds for all $k \geq 1$ as soon as it holds for $k=1$.

Proof. We analyze the Markov chain transition probabilities $P^{(n)}$. In Section 2 we used a distribution function $F \equiv F^{(n)}$ to define $P^{(n)}$. Let $\varepsilon_{n}, n \geq 1$, be independent random variables having distribution $F^{(n)}, n \geq 1$. Define

$$
\begin{aligned}
Z_{0}^{(i)} & :=i, & & i \geq 0, \\
Z_{n}^{(i)} & :=0, & & \text { if } Z_{n-1}^{(i)}=0, \\
& :=\max \left(Z_{n-1}^{(i)}-1, \varepsilon_{n}\right), & & \text { otherwise. }
\end{aligned}
$$


It is easily seen that $\left(Z_{n}^{(i)}\right)_{n \geq 0}$ is distributed as the Markov chain $\left(Z_{n}\right)_{n \geq 0}$ started at $\{i\}$ and also that $Z_{n}^{(i)}$ is monotone in $i \geq 0$. All of the $Z^{(i)}$-processes are given in terms of $\left(\varepsilon_{1}, \varepsilon_{2}, \ldots\right)$. Because $\{0\}$ is absorbing for the Markov chain the event $A_{k}:=\left\{Z_{k}^{(1)} \geq 1\right\}$ can be written as $A_{k}=\bigcap_{1 \leq t \leq k}\left\{Z_{t}^{(1)} \geq 1\right.$ for $1 \leq t \leq$ $k\}$. Also $A_{k}$ is increasing in $\left(\varepsilon_{1}, \varepsilon_{2}, \ldots\right)$. It has positive probability by assumption and by the FKG-inequality

$$
P\left(Z_{n}^{(k)} \geq j \mid A_{k}\right) \geq P\left(Z_{n}^{(k)} \geq j\right) .
$$

On the set $A_{k}, Z_{t}^{(k)} \geq Z_{t}^{(1)} \geq 1$ holds for $1 \leq t \leq k$. If on $A_{k}$ for all $1 \leq t \leq k$ one has that $Z_{t}^{(k)}=Z_{t-1}^{(k)}-1$ holds, then $Z_{k}^{(k)}=Z_{0}^{(k)}-k=0$, contradicting $Z_{k}^{(k)} \geq 1$. Hence for some $1 \leq t \leq k$ we have $Z_{t}^{(k)}=\varepsilon_{t}>Z_{t-1}^{(k)}-1$ but then also $Z_{t}^{(1)}=\varepsilon_{t}$. So on $A_{k}$ there is some $1 \leq t \leq k$ such that $Z_{t}^{(k)}=Z_{t}^{(1)}$ and then $Z_{n}^{(k)}=Z_{n}^{(1)}$ for all $n \geq t$. Hence on $A_{k} Z_{n}^{(k)}=Z_{n}^{(1)}$ holds for $n \geq k$. Thus (3.5) becomes for $n \geq k$,

$$
P_{1}\left(Z_{n} \geq j \mid Z_{k} \neq 0\right) \geq P_{k}\left(Z_{n} \geq j\right) .
$$

Using this with $j=1$ and assuming (3.2) for $k=1$ we get (3.2) for any $k \geq 1$.

Define the $\psi$-mixing coefficient $\psi(\mathscr{A}, \mathscr{B})$ between $\sigma$-fields $\mathscr{A}$ and $\mathscr{B}$ as

$$
\psi(\mathscr{A}, \mathscr{B})=\sup \left|\frac{\mu(A \cap B)}{\mu(A) \mu(B)}-1\right|,
$$

where the supremum is taken over $A \in \mathscr{A}$ and $B \in \mathscr{B}$ having positive measure. This coefficient dominates the correlation between $1_{A}$ and $1_{B}$. Our inequality gives the following.

CoRollary 3.4. If $\mu$ is any Gibbs measure for $\Delta H$, then

$$
\psi\left(\mathscr{B}_{\Lambda_{k}}, \mathscr{B}_{\bar{\Lambda}_{N}}\right) \leq P_{k}(\tau \leq N)^{-2}-1 .
$$

Proof. The measure $\nu:=\mu(\cdot \mid B)$ with $B \in \mathscr{B}_{\bar{\Lambda}_{N}}$ satisfies, for $\Lambda=\Lambda_{N}$,

$$
\nu\left(d \sigma_{\Lambda}, d \sigma_{\bar{\Lambda}}\right)=\frac{1}{\mu(B)} \rho_{\Lambda, \sigma}\left(d \sigma_{\Lambda}\right) 1_{B}\left(\sigma_{\bar{\Lambda}}\right) \mu_{\bar{\Lambda}}\left(d \sigma_{\bar{\Lambda}}\right)=\rho_{\Lambda, \sigma}\left(d \sigma_{\Lambda}\right) \nu_{\bar{\Lambda}}\left(d \sigma_{\bar{\Lambda}}\right),
$$

where $\rho_{\Lambda, \sigma}$ depends only on $\sigma_{\bar{\Lambda}}$ as we noted in Section 1. By Lemma 3.1,

$$
d \leq \frac{\nu(A)}{\mu(A)} \leq d^{-1},
$$

where the last inequality was obtained by interchanging $\mu$ and $\nu$, and $d=$ $P_{k}(\tau \leq N)^{2}$. Hence $\mu(A \cap B) / \mu(A) \mu(B)$ is between $d$ and $d^{-1}$ uniformly and this easily implies the assertion.

REMark 3.5. If $\mu$ is a Gibbs measure for $\Delta H$ and (3.2) holds for all $k$, then the relevant $\psi$-mixing coefficient above vanishes asymptotically as $N \rightarrow \infty$. If, 
e.g., $S=\mathbb{Z}$ and $\Delta H$ is invariant under the translation $T$ it follows that the dynamical system $\left(X^{\mathbf{Z}}, \mu, T\right)$ is a $K$-system.

4. The simple Markov chain. The inhomogeneous chain $\left(Z_{n}\right)$ of key Lemma 3.1 is studied here further. A comparison with a simpler homogeneous chain $\left(Z_{n}^{*}\right)$ gives us the proof of Theorem 1.1. The use of the inhomogeneous chain directly is more complicated. In Remark 6.1 we show by an example that this may give a better result. At the end of this section we give a calculation concerning the inhomogeneous chain.

Let $Z_{n}$ be the Markov chain with transition matrices $P^{(1)}, P^{(2)}, \ldots$ where

$$
P^{(n)}=\left(\begin{array}{cccc}
1 & 0 & 0 & \cdots \\
p_{0}^{(n)} & p_{1}^{(n)}-p_{0}^{(n)} & p_{2}^{(n)}-p_{1}^{(n)} & \ldots \\
0 & p_{1}^{(n)} & p_{2}^{(n)}-p_{1}^{(n)} & \ldots \\
0 & 0 & p_{2}^{(n)} & \ldots \\
\cdot & \cdot & \cdot & \ldots
\end{array}\right)
$$

as in the preceding section. Here $\{0\}$ is an absorbing state;

$$
p_{k}^{(n)}:=\exp \left(-\operatorname{var}_{k}\left(\phi_{n}\right)\right)
$$

We want to investigate whether $P_{k}\left(Z_{n}=0\right) \uparrow 1$ as $n \rightarrow \infty$. By Proposition 3.3 this in general only has to be done for $k=1$ because of the special structure of the chain. We will now derive (4.7) concerning $\Delta_{n}:=P_{1}\left(Z_{n} \neq 0\right), n \geq 0$, where $\Delta_{n}$ is nonincreasing.

Write for $k \geq 0$,

$$
G_{n}(k):=P_{1}\left(Z_{n} \leq k\right) \text { and } g_{n}(k):=P_{1}\left(Z_{n}=k\right) .
$$

Then from $g_{n}=g_{n-1} P^{(n)}, n \geq 1$,

$$
G_{n}(k)=G_{n-1}(0)\left(1-p_{k}^{(n)}\right)+G_{n-1}(k+1) p_{k}^{(n)}
$$

follows, as an easy calculation shows. Write $\Delta_{n}(k)=1-G_{n}(k)$. We can calculate inductively from

$$
\Delta_{n}(k)=\Delta_{n-1}\left(1-p_{k}^{(n)}\right)+\Delta_{n-1}(k+1) p_{k}^{(n)}
$$

that

$$
\begin{aligned}
\Delta_{n}(0)= & \Delta_{n-1}\left(1-p_{0}^{(n)}\right)+\Delta_{n-1}(1) p_{0}^{(n)} \\
= & \Delta_{n-1}\left(1-p_{0}^{(n)}\right)+\Delta_{n-2}\left(p_{0}^{(n)}-p_{0}^{(n)} p_{1}^{(n-1)}\right) \\
& +\Delta_{n-2}(2) p_{0}^{(n)} p_{1}^{(n-1)}
\end{aligned}
$$

We continue to apply (4.2) in this way to get

$$
\Delta_{n}=\Delta_{n-1} H^{(n)}\{1\}+\Delta_{n-2} H^{(n)}\{2\}+\cdots+\Delta_{0} H^{(n)}\{n\} .
$$

Here we use that $\Delta_{0}(0)=1$ and $\Delta_{0}(1)=\Delta_{0}(2)=\cdots=0$ and furthermore that 
$H^{(n)}$ is a probability measure such that

$$
H^{(n)}\{k\}=h_{k-1}^{(n)}-h_{k}^{(n)} \quad \text { where } \quad h_{k}^{(n)}:=p_{0}^{(n)} \cdots p_{k-1}^{(n-(k-1))}
$$

for $1 \leq k \leq n, n \geq 1$ and $h_{0}^{(n)}:=1$.

We now reformulate this into a description using random variables. Assume that $H^{(n)}, n \geq 1$, is a probability measure on $\{1,2,3, \ldots\}$ defined arbitrarily on $\{n+1, n+2, \ldots\}$. Let $Y^{(n)}$ be independent random variables distributed as $H^{(n)}$. Then (4.4) can be summarized as

$$
\Delta_{n}=E \Delta_{n-Y^{(n)}, \quad n \geq 1,}
$$

if we define $\Delta_{n}=0$ for $n<0$. For $n=0$ we have $\Delta_{n}=1$ and if we take $Y^{(n)} \equiv 0$ for all $n \leq 0$, then (4.6) is valid for all integers $n$. To investigate $\Delta_{n}$ for fixed $n \geq 0$ we study the following random walk with space inhomogeneous independent increments:

$$
\sigma_{0}:=n, \quad \sigma_{k+1}:=\sigma_{k}-Y^{\left(\sigma_{k}\right)}, \quad k \geq 0 .
$$

This random walk stops on $\{0,-1, \ldots\}$ and 0 is the only element in this set with nonvanishing $\Delta$-value. One now easily observes that

$$
\Delta_{n}=E \Delta_{\sigma_{1}}=\cdots=P_{\{n\}}\left(\text { the random walk } \sigma_{k} \text { hits }\{0\}\right) .
$$

Theorem 1.1 will follow by using renewal theory to study (4.7) and (4.4).

Proof of Theorem 1.1. We want to apply Corollary 3.2. Let $P^{(n)}$ be associated to $Z_{n}$ as above with $p_{k}^{(n)}=\exp \left(-\operatorname{var}_{k}\left(\phi_{n}\right)\right)$ by our definitions at the end of Section 2 and in Section 3. We compare $Z_{n}$ with a time homogeneous Markov chain $Z_{n}^{*}$ as follows. For all $n \geq 1$ replace in the definition of $P^{(n)}$ the value of $p_{k}^{(n)}$ by

$$
p_{k}^{*}:=\inf _{n \geq 1} p_{k}^{(n)}=\exp \left(-r_{k}\right)
$$

with $r_{k}$ as in Section 1 . Then if $k \geq m$ we have

$$
\sum_{i \geq j} P_{k i}^{(n)} \leq \sum_{i \geq j} P_{m i}^{*}
$$

One uses this and induction on $n$ to get $P_{k}\left(Z_{n} \geq j\right) \leq P_{k}\left(Z_{n}^{*} \geq j\right)$ for all $k, n \geq 1$ with $Z_{n}^{*}$ the Markov chain with transition probability $P^{*}$. By Corollary 3.2 it is sufficient to prove $P_{k}\left(Z_{n} \neq 0\right) \downarrow 0$ as $n \rightarrow \infty$ and clearly this follows from $P_{k}\left(Z_{n}^{*} \neq 0\right) \downarrow 0$ as $n \rightarrow \infty$. Thus by Proposition 3.3 it is sufficient to prove $\Delta_{n}^{*}:=$ $P_{1}\left(Z_{n}^{*} \neq 0\right) \downarrow 0$ as $n \rightarrow \infty$. Now take the random walk $\left(\sigma_{k}^{*}\right)_{k \geq 0}$ as the analogue of $\left(\sigma_{k}\right)_{k \geq 0}$ above. It can be chosen to have increments distributed as $H^{*}$ (thus space homogeneous) on the positive integers and by (4.4) we have

$$
\Delta_{n}^{*}=\Delta^{*} \otimes H^{*}, \quad n \geq 1,
$$

where $\otimes$ denotes convolution. Then $\left(\Delta_{n}^{*}\right)_{n \geq 0}$ is well known in probability theory 
to be a renewal sequence and by Erdös, Feller and Pollard (1949),

$$
\lim _{n \rightarrow \infty} \Delta_{n}^{*}=\frac{1}{\mu},
$$

where $\mu$ is the mean of $H^{*}$, i.e., equals

$$
\sum_{n \geq 1} n H^{*}(\{n\})=\sum_{n \geq 1} n\left(p_{0}^{*} \cdots p_{n-2}^{*}-p_{0}^{*} \cdots p_{n-1}^{*}\right)=\sum_{n \geq 0} p_{0}^{*} \cdots p_{n-1}^{*},
$$

so is infinite by assumption (1.3). So $\Delta_{n}^{*} \downarrow 0$ as $n \rightarrow \infty$ as was to be proved.

Remark 1.3 follows using the argument above and the assertion about weakening (3.2) made in Section 3.

REMARK 4.1. In considering the proof above (and also $L$ of Section 3 and its associated Markov operator) the role of duality is in the background. We investigate the process $\left(Z_{n}^{*}\right)$ constructed above. The existence of a dual Markov process $Z^{\sim}$ such that for $i, j \geq 0$,

$$
P\left(Z_{n}^{*} \geq j \mid Z_{0}^{*}=i\right)=P\left(Z_{n}^{-} \leq i \mid Z_{0}^{-}=j\right),
$$

can be studied as in Siegmund (1976). It is seen that it exists because $\left(Z_{n}^{*}\right)$ is stochastically monotone in the sense that the left-hand side of (4.8) is nondecreasing in $i$. The transition matrix $P^{-}$of $Z^{\sim}$ is seen to be

$$
\left(\begin{array}{cccccc}
1 & 0 & 0 & 0 & 0 & \cdots \\
0 & 1-p_{0}^{*} & p_{0}^{*} & 0 & 0 & \cdots \\
0 & 1-p_{1}^{*} & 0 & p_{1}^{*} & 0 & \ldots \\
0 & 1-p_{2}^{*} & 0 & 0 & p_{2}^{*} & \ldots \\
. & \cdot & \cdot & \cdot & \cdot & \ldots
\end{array}\right) .
$$

Now consider $\left\{n \geq 0: Z_{n+1}^{-}=1\right\}$ as "renewal epochs" [see Feller (1966)], while $Z_{n}^{-}$is a "backward recurrence time," measuring the time lapse since the preceding renewal epoch. Berbee (1987) contains a uniqueness theorem with similar conditions but with a rather different proof than Theorem 1.1, using the process $Z^{\sim}$ instead of $Z^{*}$. Duality seems to play a role in the analogy.

The proof above used comparison with a homogeneous chain. However using the inhomogeneous chain directly may give a better bound for the critical temperature. In Remark 6.1 we indicate this using the following analysis to be applied for the inhomogeneous chain. Because this chain $Z_{n}$ has absorbing state $\{0\}$ we know $\Delta_{n}$ is nonincreasing. We may write $\Delta_{n}=1-\left(\delta_{1}+\cdots+\delta_{n}\right)$ with all $\delta_{n} \geq 0$.

Lemma 4.2. Define

$$
\psi_{n}(s):=\sum_{k \geq 0} h_{k}^{(n+k)} s^{k}, \quad 0 \leq s<1 .
$$


Then, assuming $s^{m} \psi_{m}(s) \rightarrow 0$ as $m \rightarrow \infty, 0 \leq s<1$, we have

$$
\psi_{0}(s)=1+\sum_{k \geq 1} s^{k} \psi_{k}(s) \delta_{k} .
$$

Observe that $\Delta_{n} \downarrow 0$ is equivalent to showing $\sum_{k \geq 1} \delta_{k}=1$.

Proof. Define $\Delta(s)=\sum_{n \geq 0} \Delta_{n} s^{n}$. Using (4.4) we have

$$
\Delta(s)=\Delta_{0}+\sum_{n \geq 1} \sum_{1 \leq k \leq n} \Delta_{n-k}\left(h_{k-1}^{(n)}-h_{k}^{(n)}\right) s^{n} .
$$

Write $s^{n}=s^{n-k} s^{k}$, exchange summation (using $s<1$ ) and write $m=n-k$ to get

$$
\Delta(s)=1+\sum_{k \geq 1} \sum_{m \geq 0} \Delta_{m} s^{m}\left(h_{k-1}^{(m+k)}-h_{k}^{(m+k)}\right) s^{k} .
$$

Using (4.9) a simple calculation gives

$$
1=\sum_{m \geq 0} \Delta_{m}\left(s^{m} \psi_{m}(s)-s^{m+1} \psi_{m+1}(s)\right) .
$$

Because $\Delta_{n}$ is increasing $\Delta_{n}=1-\delta_{1}-\cdots-\delta_{n}$ with all $\delta_{n} \geq 0$. Substituting this in the equality above and using telescoping sums and exchange of summation, one finds the assertion.

5. A Perron-Frobenius theorem. We study a positive operator and indicate at the end of this section an important difference with the Perron-Frobenius theorem of Ruelle (1968). The proof of Theorem 1.1 is followed closely.

Our Perron-Frobenius theorem can be described as follows. Let $X$ be a finite set and $S=\{1,2, \ldots\}$. We construct an operator $L: \mathscr{M}\left(X^{S}\right) \rightarrow \mathscr{M}\left(X^{S}\right)$ by defining

$$
L \mu(B):=\sum_{\sigma_{0} \in X} \int_{X^{S}} \exp \left(\phi\left(\left(\sigma_{0}, x\right)\right)\right) I_{B}\left(\left(\sigma_{0}, x\right)\right) \mu(d x),
$$

where $\left(\sigma_{0}, x\right)$ is seen as an element of $X^{S}$ and $\phi$ is a real function on $\Pi_{n \geq 0} X$. Using the notation of Section 1 we have the following.

THEOREM 5.1. If

$$
\sum_{k \geq 0} \exp \left(-\operatorname{var}_{0}(\phi)-\cdots-\operatorname{var}_{k}(\phi)\right)=\infty,
$$

then there is a unique probability measure $\nu$ with $L \nu=\lambda \nu$ for some $\lambda>0$ and for any other bounded measure $\mu$ and any cylinder set $C \subset X^{S}$,

$$
\begin{aligned}
& L^{n} \mu(C) \sim\left\|L^{n} \mu\right\| \nu(C), \\
& \frac{\left\|L^{n+1} \mu\right\|}{\left\|L^{n} \mu\right\|} \rightarrow \lambda \text { as } n \rightarrow \infty .
\end{aligned}
$$


Proof. Using the argument in the proof of Theorem 1.1 we find that

$$
\left(\frac{\rho_{-k}\left(L^{n} \mu, L^{n} \nu\right)}{\rho_{k}\left(L^{n} \mu, L^{n} \nu\right)}\right)^{1 / 2} \geq P_{k}(\tau \geq n),
$$

where $\tau$ is the absorption time into $\{0\}$ of a Markov chain $Z_{n}^{*}, n \geq 0$, with transition probabilities $P$ associated at the end of Section 2 with the operator $\exp (\phi)$. Again as in the proof of Theorem 1.1 (or using Lemma 4.2 and Proposition 3.3) the right-hand side of (5.3) is asymptotically 1 as $n \rightarrow \infty$ and we easily get

$$
\frac{L^{n} \mu(C)}{L^{n} \nu(C)} \sim \frac{\left\|L^{n} \mu\right\|}{\left\|L^{n} \nu\right\|} .
$$

Existence of $\nu$ such that $L \nu=\lambda \nu$ follows by finding a fixed point of the operator $\tilde{L}$ defined by $\tilde{L} \mu:=L \mu /\|L \mu\|$. Such a fixed point can be obtained as the limit of a convergent subsequence of

$$
\frac{1}{n} \sum_{k=1}^{n} \tilde{L}^{k} \mu, \quad n \geq 1
$$

because $X^{S}$ is compact. Uniqueness of such a $\nu$ follows from (5.4). Moreover (5.2) is also implied by (5.4).

Above we obtained an "eigenmeasure" $\nu$ of $L$ as any normalized limit of $L^{n} \mu$, $n \geq 1$. In Ruelle (1968) there is also constructed an eigenfunction $h$ at eigenvalue $\lambda$ for the adjoint $L^{*}$, that satisfies

$$
\frac{L^{n} \delta_{\{x\}}}{L^{n} \delta_{\{y\}}} \sim \frac{h(x)}{h(y)} \quad \text { as } n \rightarrow \infty .
$$

The extension we give here is interesting because our context seems more sensitive: It may be that (5.5) does not have to hold if one merely assumes (5.1).

6. A one-dimensional Ising model with long range interaction. A nice aspect of our results is that only little structure is presupposed. Also we hope our approach may be useful for, e.g., spin glasses and the rotator model. Our application here has much structure: It has pair interactions and is one-dimensional. To describe it let $X:=\{-1,1\}$ and $S=\mathbb{Z}$. Assume for finite $\Lambda \subset \mathbb{Z}$,

with

$$
\Delta H\left(\sigma, \sigma^{\prime}\right)=\phi_{\Lambda}\left(\sigma^{\prime}\right)-\phi_{\Lambda}(\sigma) \text { if } \sigma_{\bar{\Lambda}}=\sigma^{\prime}{ }_{\bar{\Lambda}}
$$

$$
\phi_{\Lambda}(\sigma)=-\frac{1}{2} \beta \sum J(|j-i|)\left(\sigma_{i} \sigma_{j}-1\right),
$$

where the sum is over $i<j$ with $i, j \in \mathbb{Z}^{2} \backslash(\bar{\Lambda} \times \bar{\Lambda})$. We consider first $J \geq 0$.

We study $\beta_{c}$, the maximum of all $\beta \geq 0$ below which there is a unique Gibbs measure. If $J(n)=1 / n^{\alpha}$ and $\alpha>2$, then by Ruelle (1968) there is a unique Gibbs measure at any temperature, i.e., $\beta_{c}=\infty$. On the other hand Dyson (1969a) showed $0<\beta_{c}<\infty$ for $1<\alpha<2$. Fröhlich and Spencer (1982) suc- 
ceeded in proving this also for $\alpha=2$. We investigate the boundary case and prove

$$
\beta_{c} \geq \liminf \frac{\log n}{4 \sum_{1 \leq k \leq n} k J(k)} .
$$

Write $a(n)=o(b(n))$ if $a(n) / b(n) \rightarrow 0$ as $n \rightarrow \infty$. By (6.1) we have $\beta_{c}=\infty$ if $S_{n}:=\sum_{1 \leq k \leq n} k J(k)=o(\log n)$ and note also that the lower bound on $\beta_{c}$ is positive if $J(n)=1 / n^{2}$ which makes this result seem natural. Dyson (1969b) proved earlier that $\beta_{c}=\infty$ if $S_{n}=o(\log \log n)$ and Rogers and Thompson (1981) improved this to $S_{n}=o\left((\log n)^{1 / 2}\right)$. Fannes, Vanheuverzwijn and Verbeure (1982) obtained $\beta_{c}=\infty$ with our rate on $S_{n}$ but assuming, however, polynomial decay of spin correlations. The important recent paper of Aizenman, Chayes, Chayes and Newman (1988) will be discussed at the end of this section.

To derive (6.1) about uniqueness of Gibbs measures we use Theorem 1.1. Take $\Lambda_{0}:=\varnothing, \Lambda_{n}:=\{-n+1, \ldots, n\}, n \geq 1$, and consider $\phi_{n}$ defined by (1.2) which can be written as

$$
\phi_{n}(\sigma)=-\frac{1}{2} \beta \sum J(j-i)\left(\sigma_{i} \sigma_{j}-1\right),
$$

where the sum is over all $i<j$ such that $(i, j) \in\left(\bar{\Lambda}_{n-1} \times \bar{\Lambda}_{n-1}\right) \backslash\left(\bar{\Lambda}_{n} \times \bar{\Lambda}_{n}\right)$. A simple calculation gives for $k \geq 0$,

$$
\operatorname{var}_{k}\left(\phi_{n}\right)=2 \beta\left[\sum_{i>k} J(i)+\sum_{i>2 n+k-1} J(i)\right] .
$$

Hence we have

$$
r_{k} \leq 4 \beta \sum_{i>k} J(i)
$$

By Theorem 1.1 there is a unique Gibbs measure if for $n$ large,

$$
\exp \left(-r_{0}-\cdots-r_{n-1}\right) \geq \frac{1}{n},
$$

or equivalently if

$$
\beta \leq \frac{\log n}{4 T_{n}},
$$

where $T_{n}=\sum_{0 \leq k<n} \sum_{i>k} J(i)$. This implies (6.1) because

$$
\lim \sup \frac{1}{\log n} S_{n}=\limsup \frac{1}{\log n} T_{n}
$$

This equality is seen as follows. Observe that $\leq$ holds because, using exchange of summation in $T_{n}$, one finds $S_{n} \leq T_{n}$. The opposite inequality is easily derived by using $(1 / n) T_{n}=\sum_{k \geq n}[1 /(k(k+1))] S_{k}$ together with $\sum_{k \geq n}[1 /(k(k+1))] \log k \sim(1 / n) \log n$. Thus the proof of $(6.1)$ is completed.

Let us now give some criticism. If $J(n)=1 / n^{\alpha}$ with $1<\alpha<2$, then (6.1) reduces to the trivial bound $\beta_{c} \geq 0$. So our result is curiously sensitive near $\alpha=2$ but is insensitive for smaller $\alpha$. Fröhlich and Spencer (1982) mention a 
correlation inequality from which it seems our result cannot be derived. However at $\alpha=2$ use of this inequality gives a better result than we get from Corollary 3.4 for spin correlation asymptotics. As is illustrated in Remark 4.1, the comparison we make in our method is related to a renewal process, which is one of the simplest one-dimensional random processes. We hope that using other random processes may give better results. It would be interesting if the bound in Dobrushin (1968) could be included in an improved comparison result.

Let us also mention that in case we allow $J$ also to be negative, one has (6.1) but with $J(k)$ replaced by $|J(k)|$. For this the argument above can be followed with this replacement but (6.2) becomes an inequality. In special cases one can do better however by taking a different choice of $\Lambda_{n}$. Because this would lead to too technical an analysis we do not discuss it here.

REMARK 6.1. Using Tauberian theory we show for the case $J(n)=1 / n^{2}$ that the inhomogeneous chain of our method gives better bounds than the homogeneous chain. To know this may be of value for our method. We succeed to improve $\beta_{c} \geq \frac{1}{4}$ to $\beta_{c} \geq \frac{1}{2}$. A clue to this improvement is that the second term in (6.2) does not seem to play a role for large $n$. In Section 3 we noted in this connection that individual $\phi_{n}$ do not play a role in our bound but the improvement here goes further. By (4.5) and (4.4) we find

$$
h_{k}^{(n+k)}=\exp \left(-2 \beta\left[x_{1}+\cdots+x_{k}+x_{2 n+k+1}+\cdots+x_{2 n+2 k}\right]\right),
$$

where $x_{j}=\sum_{i \geq j} J(i)$. As $n \rightarrow \infty$ we have $h_{k}^{(n+k)} \uparrow h_{k}^{(\infty)}$. With the obvious notation we find from Lemma 4.2 ,

$$
\frac{\psi_{0}(s)}{\psi_{\infty}(s)}=\frac{1}{\psi_{\infty}(s)}+\sum_{k \geq 1} s^{k} \frac{\psi_{k}(s)}{\psi_{\infty}(s)} \delta_{k} .
$$

Because $\psi_{k} / \psi_{\infty} \leq 1$ and $\sum_{k \geq 1} \delta_{k} \leq 1$ we may apply the bounded convergence theorem. Using Feller (1966), Theorem 13.5.5, one finds for $\beta=\frac{1}{2}$ that

$$
\frac{\psi_{k}(s)}{\psi_{\infty}(s)} \sim \frac{1}{2}
$$

as $s \uparrow 1$. Because $\psi_{\infty}(1)=\infty$ we find $\sum_{k \geq 1} \delta_{k}=1$ and $\Delta_{n} \downarrow 0$. Thus $\beta_{c} \geq \frac{1}{2}$. If we study in the last result $S=\mathbb{N}$ instead of $S=\mathbb{Z}$ we get $\beta_{c} \geq 1$. We note however that Aizenman, Chayes, Chayes and Newman (1988) discussed below would give in both cases $\beta_{c} \geq 1$ which is clearly better for $S=\mathbb{Z}$. An explanation for this is possibly that we measure interaction via $\exp \left(-\operatorname{var}_{k}\left(\phi_{n}\right)\right)$ in (4.1): We hardly use the fact that there is no strong direct interaction between $\sigma_{n}$ and $\sigma_{-n}$.

For the revision of this paper the results of Aizenman, Chayes, Chayes and Newman (1988) were available. The paper proves the existence of a jump in the magnetization at $\beta_{c}$. It appears that the connection of this (Thouless-) jump with uniqueness results as above is close because it is obtained by a further study of a uniqueness result. This result is that $\beta_{c} \geq \liminf \left(n^{2} / J(n)\right)$, which resembles, e.g., (6.1). Our method was found in 1985 before news about the existence of a proof of the Thouless-jump reached the author. Its approach using the 
Perron-Frobenius operator and ideas related to Berbee (1987) seems quite different. The approach of Aizenman, Chayes, Chayes and Newman (1988) uses more structure: In a nice probabilistic analysis it connects via the Fortuin-Kasteleyn representation for Potts models the uniqueness problem to a percolation problem solved in Aizenman and Newman (1986), also using a Markov chain.

\section{REFERENCES}

Aizenman, M., Chayes, J. T., Chayes, L. and Newman, C. M. (1988). Discontinuity in one-dimensional $1 /|x-y|^{2}$ Ising and Potts models. J. Statist. Phys. $501-40$.

Aizenman, M. and Newman, C. M. (1986). Discontinuity of the percolation density in one-dimensional $1 /|x-y|^{2}$ percolation models. Comm. Math. Phys. 107 611-647.

BerbeE, H. (1987). Chains with infinite connections: Uniqueness and Markov representation. Probab. Theory Related Fields 76 243-253.

Bowen, R. (1975). Equilibrium States and the Ergodic Theory of Anosov Diffeomorphisms. Lecture Notes in Math. 470. Springer, New York.

Dobrushin, R. L. (1968). The problem of uniqueness of a Gibbsian random field and the problem of phase transition. Functional Anal. Appl. 2 302-312.

Dyson, F. J. (1969a). Existence of a phase-transition in a one-dimensional Ising ferromagnet. Comm. Math. Phys. 12 91-107.

Dyson, F. J. (1969b). Nonexistence of spontaneous magnetization in a one-dimensional Ising ferromagnet. Comm. Math. Phys. 12 212-215.

Erdös, P., Feller, W. and Pollard, H. (1949). A theorem on power series. Bull. Amer. Math. Soc. 55 201-204.

Fannes, M., Vanheuverzwisn, P. and Verbeure, A. (1982). Energy-entropy inequalities for classical lattice systems. J. Statist. Phys. 29 547-560.

FELLER, W. (1966). An Introduction to Probability Theory and Its Applications 2. Wiley, New York.

FröHLICH, J. and SPENCER, T. (1982). The phase transition in the one-dimensional Ising model with $1 / r^{2}$ interaction energy. Comm. Math. Phys. 84 87-101.

Hofbauer, F. (1977). Examples for nonuniqueness of the equilibrium state. Trans. Amer. Math. Soc. 228 223-241.

Liggetr, T. M. (1985). Interacting Particle Systems. Springer, New York.

Rogers, J. B. and Thompson, C. J. (1981). Absence of long-range order in one-dimensional spin systems. J. Statist. Phys. 25 669-678.

Ruelle, D. (1968). Statistical mechanics of a one-dimensional lattice gas. Comm. Math. Phys. 9 267-278.

SiEGMUND, D. (1976). The equivalence of absorbing and reflecting barrier problems for stochastically monotone Markov processes. Ann. Probab. 4 914-924.

Centre for Mathematics and Computer Science

P.O. BoX 4079

1009 AB AMSTERDAM

THE NETHERLANDS 